\title{
Coming of age: the artificial pancreas for type 1 diabetes
}

\author{
Hood Thabit ${ }^{1,2} \cdot$ Roman Hovorka ${ }^{1,3}$
}

Received: 16 March 2016/Accepted: 24 May 2016/Published online: 30 June 2016

(C) The Author(s) 2016. This article is published with open access at Springerlink.com

\begin{abstract}
The artificial pancreas (closed-loop system) addresses the unmet clinical need for improved glucose control whilst reducing the burden of diabetes self-care in type 1 diabetes. Glucose-responsive insulin delivery above and below a preset insulin amount informed by sensor glucose readings differentiates closed-loop systems from conventional, threshold-suspend and predictive-suspend insulin pump therapy. Insulin requirements in type 1 diabetes can vary between one-third-threefold on a daily basis. Closed-loop systems accommodate these variations and mitigate the risk of hypoglycaemia associated with tight glucose control. In this review we focus on the progress being made in the development and evaluation of closed-loop systems in outpatient settings. Randomised transitional studies have shown feasibility and efficacy of closed-loop systems under supervision or remote monitoring. Closed-loop application during free-living, unsupervised conditions by children, adolescents and adults compared with sensor-augmented pumps have shown improved glucose outcomes, reduced hypoglycaemia and positive user acceptance. Innovative approaches to enhance closed-loop performance are discussed and we also present the outlook and strategies used to ease clinical adoption of closed-loop systems.
\end{abstract}

Roman Hovorka

rh347@cam.ac.uk

1 Wellcome Trust-MRC Institute of Metabolic Science, University of Cambridge, Level 4, Institute of Metabolic Science, Box 289, Addenbrooke's Hospital, Hills Rd, Cambridge CB2 0QQ, UK

2 Department of Diabetes \& Endocrinology, Cambridge University Hospitals NHS Foundation Trust, Cambridge, UK

3 Department of Paediatrics, University of Cambridge, Cambridge, UK
Keywords Artificial pancreas · Closed-loop system · Continuous glucose monitor · Control algorithm · Insulin pump $\cdot$ Review $\cdot$ Type 1 diabetes

\section{Introduction}

Since the late 1960s, when capillary blood glucose meters were introduced into clinical practice [1], the progress in diabetes management has become intrinsically linked to innovations in diabetes technology. Insulin pump therapy, the clinical feasibility of which was established in the 1970s $[2,3]$, is an increasingly applied treatment modality, particularly in the paediatric population. This approach uses smart pumps with bolus calculators and data upload features to guide clinical management [4]. Minimally invasive, realtime continuous glucose measurement $[5,6]$ is progressing to accurate, insulin-dosing approved, factory-calibrated systems [7]. A concerted effort is underway to combine these advancements and develop the 'artificial pancreas', also known as the closed-loop system, to emulate the feedback, glucose-responsive functionality of the beta cell $[8,9]$.

Closed-loop systems combine real-time sensor glucose measurement with insulin pumps by using a control algorithm to direct insulin delivery (Fig. 1) [10]. The autonomous, graduated modulation of insulin delivery below and above the preset insulin amount in a glucose-responsive manner differentiates closed-loop systems from conventional insulin pump therapy and low-glucose suspend/predictive lowglucose suspend insulin delivery systems, which suspend insulin delivery when sensor glucose is at or predicted to be below a preset glucose threshold [11-13].

Closed-loop systems aim to improve glucose control whilst reducing the burden of hypoglycaemia and diabetes self-care. The clinical justification for closed-loop technology, its 
Fig. 1 A prototype closed-loop system. (a) A prototype closedloop system comprises a continuous glucose monitor (CGM) sensor and receiver, an insulin pump, and a mobile phone running a control algorithm (potentially the algorithm may be located on the insulin pump obviating the need for a hand-held controller/mobile phone device).

(b) A photo of a participant (obtained with consent) using the closed-loop system during a home study [8]

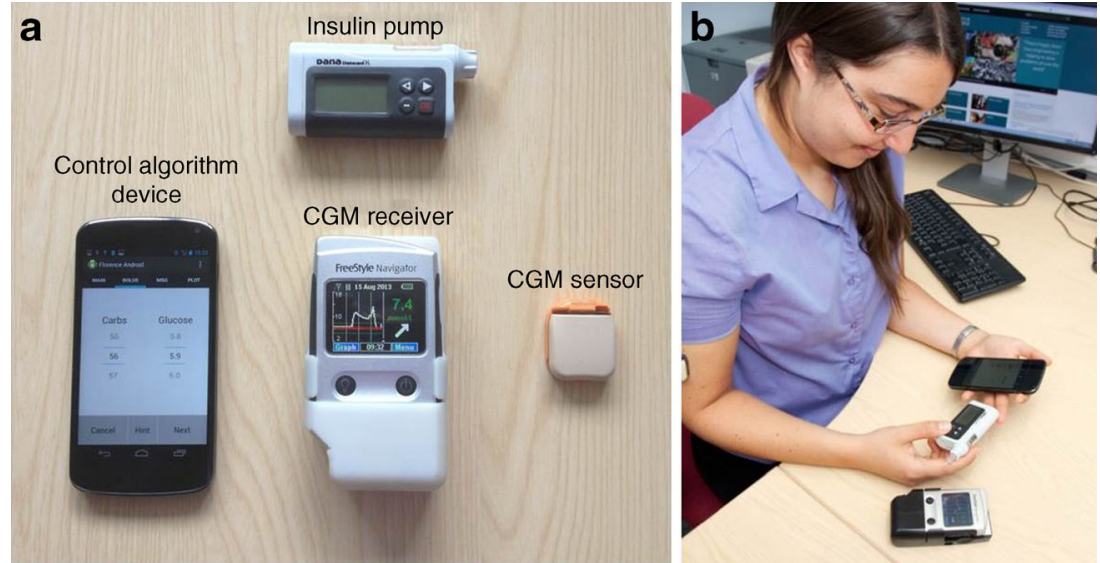

viability as a therapeutic option, findings from transitional and home studies, and the outlook and integration into clinical practice are discussed in the present review (further reviews are available $[14,15])$.

\section{Meeting the need}

Tight blood glucose control reduces the risk of long-term diabetes related complications $[16,17]$ but is limited by hypoglycaemia [18]. Insulin analogues and modern insulin regimens, including insulin pump therapy, have lowered the relative risk of hypoglycaemia compared with those observed in the intensive insulin treatment group of the transformative Diabetes Control and Complication Trial [16, 19, 20] but inherent unpredictability and variability of glucose levels remains a significant barrier. Glucose-responsive insulin delivery by closed-loop systems addresses these unmet clinical needs and aims to reduce the burden of diabetes care.

Key challenge: intra-individual variability in insulin requirements Insulin requirements vary considerably within individuals with type 1 diabetes by on average $30 \%$ overnight and $20 \%$ during waking hours. However, this can vary from one-third to three times that of planned insulin delivery, even without intercurrent illness [21]. Potential reasons include variable meal composition [22], aberrations in glucose turnover, lability due to physical activity $[23,24]$ and changes in insulin sensitivity in women during perimenstrual periods [25]; however, exact quantification and attribution is unknown. Attempts to elucidate and establish reliable insulin needs through formalised protocols or during regular clinic visits guided by data uploads $[26,27]$ are hampered by the need for frequent re-adjustments of insulin requirements. Experienced pump users may alternate basal patterns to match daily lifestyle conditions or premeditated activities [28] but this approach has variable success.
Responding to day-to-day and within-day glucose variability is the key advantage that adaptive closed-loop systems have over conventional insulin therapies. By autonomously and continually modulating insulin delivery in a glucose-responsive fashion, closed-loop systems deliver insulin to minimise hyper- and hypoglycaemic excursions. Adaptive features of the control algorithms individualise the closed-loop system to particular physiology and lifestyle patterns. The performance of closed-loop systems is, however, limited by the speed of insulin absorption and glucose sensing inaccuracies [29]. Nonetheless, via considerably variable insulin delivery, beyond that normally applied in clinical practice, the closed-loop system may help to achieve more consistent glucose levels.

User needs and expectations Type 1 diabetes carries a significant psychosocial burden and adversely impacts quality of life [30, 31]. People with type 1 diabetes and their carers demonstrate high interest and positive attitudes towards closed-loop systems $[32,33]$ and value the prospect of having 'time off from the demands of diabetes' [34].

However, the low adherence with earlier generations of continuous glucose monitors serves as a reminder of the potential fate of new technologies [35] if input from users on device complexities and form factors are not considered. This is further justified by the reported association between user adherence and diabetes technology performance [36]. Thus, health psychologists are focused on optimising future uptake and usability of closed-loop systems [37, 38].

\section{Current biological alternatives to closed-loop technology}

Whole organ pancreas and islet cell allotransplantations have been applied in clinical practice over the past 40 years $[39,40]$. Significant improvements in whole organ pancreas transplantation techniques and post-operative care have led to 
increased patient and graft survival rates in the past decade $[41,42]$. Due to the involvement of major surgery, the risk and benefit of the procedure has to be weighed carefully and on an individual basis since the rate of perioperative morbidity, mortality and re-transplantation is still substantial [43, 44]. Islet cell transplantation, on the other hand, avoids the need for major surgical intervention given that intraportal transplantation involves a less invasive percutaneous radiological procedure [39, 45]. Compared with results from whole organ pancreas transplantation, the success and durability of islet cell transplantation have been less favourable however [46]. Furthermore, the wider clinical application of islet transplantation is hampered by several obstacles including: (1) limited islet supply and limited number of clinical sites with the skills and facilities for islet preparation; (2) the drawbacks of intensive immunosuppression therapy [47]; (3) alloimmune and autoimmune attacks resulting in up to $80 \%$ islet cell loss post transplantation.

Since being introduced into clinical practice, the successful use of pancreas and islet cell transplantation as mainstay treatments for the wider population of type 1 diabetic individuals, including the very young, pregnant individuals, elderly people and those with significant comorbidities, remains limited and is unlikely to fully address the needs of the general type 1 diabetic population.

\section{Foundations of closed-loop systems}

Continuous glucose monitoring, insulin pump and control algorithms constitute backbone technologies of the closed-loop systems. These are discussed in more detail in this section.

Continuous glucose monitoring The present generation of continuous glucose monitoring devices provide a minimally invasive method to measure real-time interstitial fluid glucose levels [7]. Commercial devices use a subcutaneously implanted needle-type amperometric enzyme electrode, which measures interstitial glucose concentration by detecting changes in the electric current that is caused by the enzymecatalysed oxidation of glucose into hydrogen peroxide. Examples of the continuous glucose monitoring devices that have been used in closed-loop research include Enlite (Medtronic MiniMed, Northridge, CA, USA) [48], Dexcom G4 and G5 (Dexcom, San Diego, CA, USA) [49] and Freestyle Navigator II (Abbott Diabetes Care, Alameda, CA, USA) [8].

Generally, glucose readings are provided every 1 to 5 min for up to 7 days of continuous wear per sensor insertion. Previous generations of continuous glucose sensors were limited by inconsistent sensor accuracy compared with the reference standard (plasma glucose), with a mean relative absolute deviation of around 15-20\%. The large measurement discrepancies reported by the previous generation glucose sensors and reference methods may lead to incorrect treatment decisions [50] and adversely affect device usability and experience. In contrast, the accuracy of the latest generation of devices has improved, with the mean relative absolute deviation measuring at around $9-11 \%$ [7] which is compatible with safe operation of closed-loop systems [51].

Continuous subcutaneous insulin infusion therapy Modern insulin pumps comprise an insulin reservoir, a small batteryoperated motor (or other delivery apparatus) linked to a computerised control mechanism and a subcutaneous infusion set (cannula and tubing system) [4]. Many have a built-in, customisable bolus calculator and monitor the insulin on board', which is the amount of insulin-to-act left in the body from the previous bolus. The patch pump design (exemplified by Omnipod [Insulet, Billerica, MA, USA]) has a reservoir unit that adheres directly to the user's skin and houses an integrated infusion set and automated inserter, thus making it 'tubing-free'. Sensor-augmented insulin pumps (e.g. MiniMed Paradigm Veo [Medtronic MiniMed] and Vibe [Animas, West Chester, PA, USA]) feature integration with continuous glucose monitoring, and are associated with reductions in $\mathrm{HbA}_{1 \mathrm{c}}$ levels [52].

The sensor-augmented pump is further enhanced via 'lowglucose suspend' and 'predictive low-glucose suspend' features $[12,13,53]$. The former feature allows insulin to be automatically suspended for up to $2 \mathrm{~h}$ when sensor glucose falls below a preset threshold [11, 12], whereas the latter suspends insulin delivery when sensor glucose is predicted to be below a preset glucose threshold by use of hypoglycaemiaprediction algorithms and automatic pump suspension [13].

Control algorithms Two main families of control algorithms have been used in closed-loop clinical studies: the classic feedback proportional-integral-derivative controller [54] and the model predictive controller [55]. The classical proportional-integral-derivative controller adjusts insulin delivery by assessing departure from target glucose level (the proportional component), the area under the curve between measured and target glucose levels (the integral component), and the rate of change in the measured glucose level (the derivative component).

The model predictive approach more readily accommodates delays associated with insulin absorption and also accounts for events having a protracted influence on glucose levels, such as meals and manually delivered prandial and correction insulin boluses. A mathematical model links insulin delivery to glucose excursions and uses model-predicted glucose levels to determine optimal insulin infusion rates.

Other clinically evaluated control approaches include the fuzzy logic approach [48], which modulates insulin delivery 
on the basis of approximate rules to express empirical knowledge acquired by diabetes practitioners. Many algorithms include safety modules to constrain insulin delivery, limiting the amount of insulin on board or the maximum rate of insulin delivery, or suspending insulin delivery when glucose levels are low or decreasing.

The hybrid closed-loop approach relies on manual administration of prandial bolus to partially mitigate absorption delay of subcutaneous, rapid-acting insulin. Strategies to fully implement closed-loop systems without prandial bolus, to further reduce the burden of self-care are being considered [56, 57].

Challenges and innovations Performance of closed-loop systems is damped by variable and relatively slow absorption of currently available rapid-acting insulin analogues [58], delaying onset of and prolonging insulin action [59]. This is of particular concern during exercise and in postprandial conditions, when blood glucose fluctuations occur. Rapidacting insulin analogues, such as aspart, lispro and glulisine, achieve peak plasma insulin concentrations at approximately $0.5-2 \mathrm{~h}$, with the duration of action between $3-5 \mathrm{~h}$. These delays are compounded by the inherent $5-15 \mathrm{~min}$ lag between glucose values in the interstitial and vascular space $[60,61]$. Both the delay in insulin action and lag time for glucose transport may attenuate performance of the daytime closedloop because of the rapid fluctuations of blood glucose levels observed throughout the day (e.g. during meal times and exercise). This is reflected by greater closed-loop incremental benefits overnight, compared with daytime $[8,49]$. The advent of faster insulin aspart and other ultra-rapid insulin analogues may help to address some of these issues. In a previous study, the onset of appearance of faster insulin aspart in serum was earlier (4.9 $\mathrm{min}$ vs $11.2 \mathrm{~min}$ ) and serum faster aspart exposure was four-and-a-half times greater in the first $15 \mathrm{~min}$ postinjection, compared with standard aspart [62].

Alternative delivery routes to accelerate systemic insulin appearance include inhaled prandial insulin, which has a faster onset and shorter action profile compared with rapid-acting subcutaneous insulin [63] and preprandial administration by a closed-loop system [64], resulting in an increased amount of time spent in the target glucose range. Alternatively, a specialised heating pad may be attached to the pump infusion set to warm surrounding tissues following a prandial insulin bolus, accelerating insulin pharmacokinetics and pharmacodynamics [65].

Advances in continuous glucose monitoring technologies to improve sensor performance and user adherence include long-term (up to six months) implantable glucose sensors that are unaffected by the external sensor-signal attenuation issues faced by conventional sensors [66]. At present, a factorycalibrated subcutaneous glucose sensor can be worn for up to 2 weeks [67]. Efforts are also underway to develop a 'single-port' device, which combines sensor glucose measurements with an insulin infusion cannula into a single subcutaneous insulin infusion set [68]. Simultaneous glucose monitoring at the site of insulin infusion may help to reduce the burden of multiple set insertions by users.

\section{Clinical evaluation of closed-loop insulin delivery in transitional outpatient settings}

Clinical testing in controlled laboratory conditions has been followed by transitional studies in diabetes camps, hotels and outpatient settings. Using this research approach, participants are studied in a 'real world' environment but with close monitoring by medical and research personnel. Below, we discuss randomised controlled trials (see Table 1), although non-randomised transitional studies have also been performed [69-71].

A previous study, using 56 participants in a multicentre diabetes camp setting over a single night, was carried out to evaluate overnight closed-loop insulin delivery, with sensoraugmented pump therapy acting as control [48]. Participants were supervised and closely monitored during the study. Compared with the control therapy, the number of episodes of hypoglycaemia with sensor glucose values below $3.5 \mathrm{mmol} / \mathrm{l}$ was significantly reduced $(p=0.003)$ with overnight closed-loop insulin therapy, with comparable median glucose levels. In another study, the application of overnight closed-loop over 5-6 days in children and adolescents attending a diabetes camp did not improve the time spent in the target glucose range compared with sensor-augmented pump therapy, using an intention to treat analysis. However, time spent in the hypoglycaemic state (specified as $2.8 \mathrm{mmol} / \mathrm{l}$, $3.3 \mathrm{mmol} / \mathrm{l}$ and $3.9 \mathrm{mmol} / \mathrm{l}$ ) were reduced significantly $(p<0.03)$ [72]. In contrast, use of a hybrid day-and-night closed-loop system in a diabetes camp over 6 days showed no improvement in glucose control when compared against sensor-augmented pump therapy and low-glucose suspend approach [73].

In a multicentre randomised study at an outpatient facility, 18 participants were remotely monitored and supervised whilst using closed-loop therapy for $40 \mathrm{~h}$ [74]. Compared with sensor-augmented pump therapy, closed-loop therapy significantly reduced the risk of hypoglycaemia $(p=0.003)$ and the frequency of hypoglycaemia episodes $(p=0.02)$, although mean glucose was increased by $0.5 \mathrm{mmol} / 1$ $(p=0.04)$. In another study, overnight closed-loop use for five consecutive nights in an outpatient transitional setting resulted in significantly improved time spent within target glucose range $(p<0.001)$ and improved mean glucose levels overnight $(p<0.001)$, compared with sensor-augmented pump therapy. Improvements in overnight glucose control 


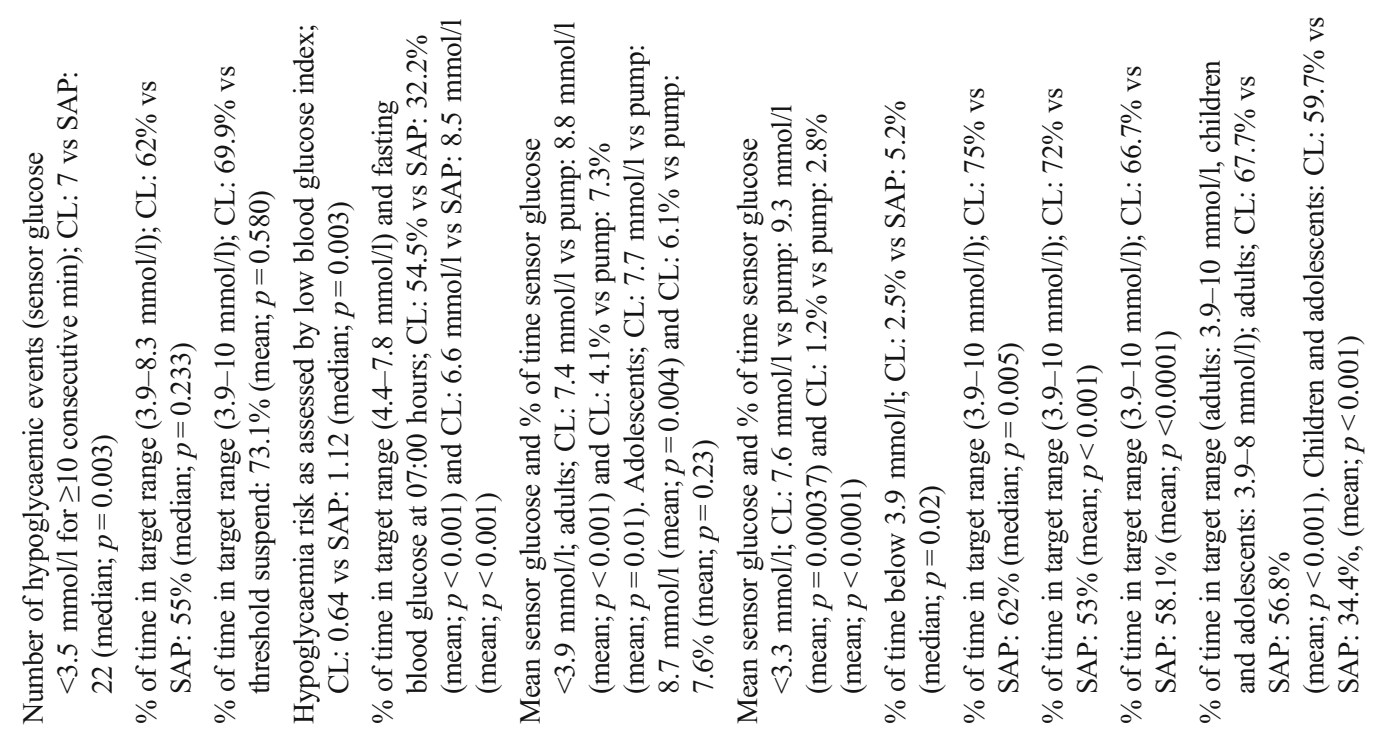

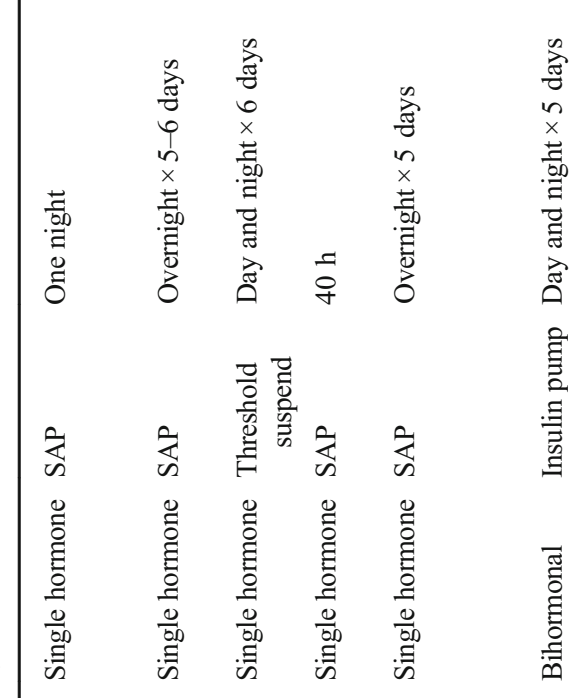

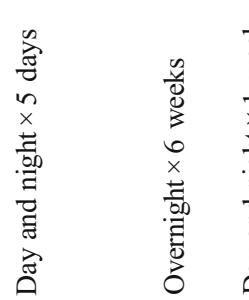

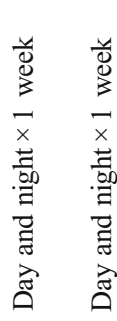

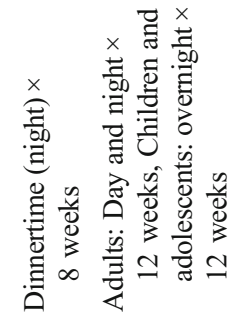

言

言

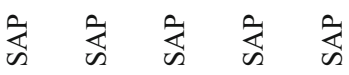

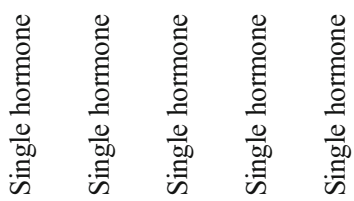

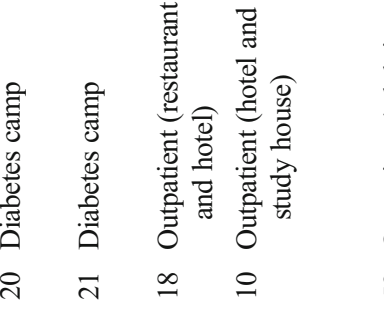

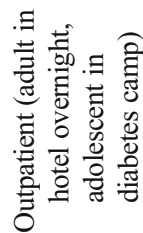

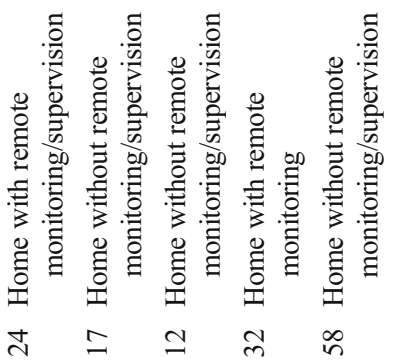

2

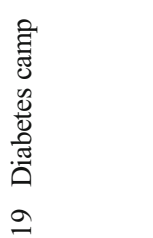

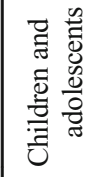

골

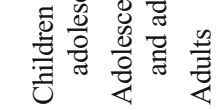

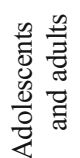

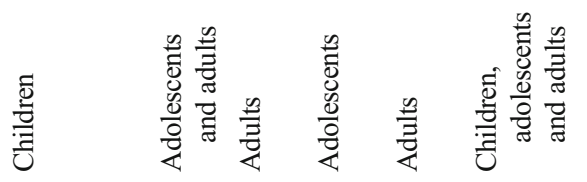

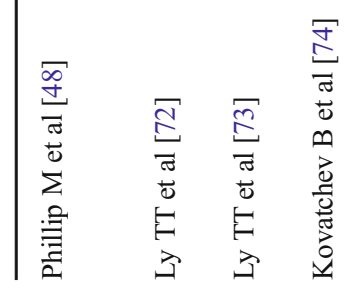

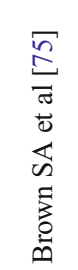

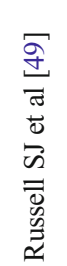

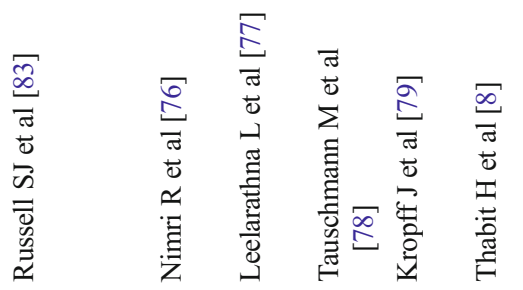

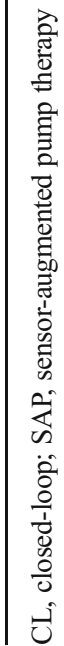


modestly correlated with daytime control $(r=0.52, p<0.01)$ [75].

\section{Home studies of closed-loop insulin delivery}

Home studies represent the final benchmark testing environment (for examples, see Table 1). However, for safety reasons, some apply remote monitoring supervision. For example, a home study with remote monitoring supervision was performed in 24 participants for 6 weeks [76]; compared with sensor-augmented pump therapy, overnight closed-loop insulin delivery resulted in a significant reduction of time spent in the hypoglycaemic state by twofold $(p=0.02)$ whilst simultaneously improving time spent within target glucose range by 11 percentage points $(p=0.003)$.

Unsupervised free-living home studies have been performed to provide unbiased assessment of closed-loop performance in the target environment. In a 1-week unsupervised day-and-night free-living closed-loop application in adults and adolescents, significant improvements in time spent within target glucose range $(p<0.01)$ and reduced mean sensor glucose levels $(p<0.03)$ were found, with no increase in time spent with hypoglycaemia $[77,78]$.

Furthermore, two multicentre free-living home studies have evaluated closed-loop systems over extended periods. In one study, participants used closed-loop insulin delivery in the evening (after dinner) and overnight for 2 months [79]. Compared with sensor-augmented insulin pump therapy, closed-loop systems improved time spent in target glucose range $(3.9-10.0 \mathrm{mmol} / \mathrm{l})$ by 8.6 percentage points $(p<0.001)$, and reduced time spent in a hypoglycaemic state $(p<0.0001)$. In addition, $\mathrm{HbA}_{1 \mathrm{c}}$ levels were also significantly reduced $(p=0.047)$. Insulin delivery during the study period was reduced whilst using the closed-loop system compared with sensor-augmented pump therapy $(p=0.029)$, and participants used the closed-loop system approximately $67 \%$ of the time between 20:00-08:00 hours.

In the second study, and the longest randomised home study to date, a 3 month, day-and-night closed-loop application in adults was compared with optimised sensoraugmented pump therapy during unrestricted free-living conditions. Closed-loop therapy improved the primary endpoint (time in target glucose range $3.9-10.0 \mathrm{mmol} / \mathrm{l}$ ) by 11 percentage points $(p<0.001)$ and reduced $\mathrm{HbA}_{1 \mathrm{c}}$ levels $(p=0.002)$ [8]. The relative risk of time spent hypoglycaemic and burden of hypoglycaemia during a $24 \mathrm{~h}$ period was reduced by closed-loop therapy compared with sensoraugmented pump therapy $(<3.9 \mathrm{mmol} / \mathrm{l} ;-19 \%, p=0.02$; and area under the curve when sensor glucose was $<3.5 \mathrm{mmol} / \mathrm{l}$; $-39 \%, p<0.001$ ). In addition, improved glucose control was achieved by closed-loop therapy, without changing total insulin delivery $(p=0.57)$. Participants used the system on their own volition $83 \%$ of the whole study duration. These results demonstrate the unique ability of closed-loop systems to simultaneously reduce mean glucose and the risk of hypoglycaemia, a feat unachievable with most other therapeutic modalities.

Apart from biomedical outcomes, qualitative studies evaluating closed-loop user feedback and experience may guide and inform future directions of closed-loop system development. In a psychosocial analysis of adult and adolescent users of overnight closed-loop systems during home studies, widely reported benefits included having 'time off' from managing their diabetes, with reduced worry of their blood glucose levels [37, 80]. Closed-loop application was found to have a positive impact on hypoglycaemia fear and other indices of health-related quality of life outcomes [38]. Common negative themes encompass device size, device connectivity and sensor calibration issues [37].

\section{Bihormonal closed-loop systems}

The risk of hypoglycaemia may be further reduced with the use of bihormonal (also known as dual-hormone) closed-loop systems delivering subcutaneous glucagon when hypoglycaemia is detected or predicted [81]. Bihormonal systems can be tuned to apply insulin in the same fashion as insulin-only closed-loop systems ('insulin-non-aggressively tuned bihormonal system') or in a more 'aggressive' fashion, anticipating that glucagon may mitigate against insulin overdelivery ('insulin-aggressively tuned bihormonal system') [82].

A day-and-night bihormonal insulin-aggressively tuned closed-loop system was studied over 5 days in a transitional outpatient setting where adult participants performed regular activities during the day and spent overnight in a hotel room while being closely supervised and monitored [49]. In addition, adolescent participants were studied in a diabetes camp setting. Overall, mean glucose was significantly reduced by $1.4 \mathrm{mmol} / \mathrm{l}(p<0.001)$ and the proportion of time spent within the target glucose range was increased $(p<0.001)$ compared with conventional pump therapy at home. Furthermore, time spent hypoglycaemic was significantly reduced in adults $(p=0.01)$. This bihormonal system delivered an average $0.8 \mathrm{mg}$ of subcutaneous glucagon per day. Another randomised crossover study evaluated bihormonal closedloop therapy in preadolescent children aged 6-11 years in an outpatient diabetes camp setting for 5 days [83]. Compared with conventional insulin pump therapy, mean sensor glucose on days $2-5$ were reduced by $1.7 \mathrm{mmol} / \mathrm{l}(p=0.0037)$ and the time spent with hypoglycaemia was also reduced $(p<0.0001)$. The bihormonal system reduced, but not completely eliminated, the need for rescue carbohydrates $(p=0.037)$. 
Mean plasma glucagon levels were projected to be above the normal fasting range.

Non-aggressive bihormonal and insulin-alone closedloop systems were compared in a paediatric diabetes camp over three consecutive nights [84]. The nocturnal time spent with hypoglycaemia with the bihormonal system was significantly reduced compared with the insulinalone system $(p=0.032)$. Mean sensor glucose levels were comparable between the two interventions.

In addition, a bihormonal fully closed-loop system (no prandial boluses) has been compared with conventional insulin pump therapy over $48 \mathrm{~h}$, in a randomised home study [85]. Median glucose levels and time spent in the target glucose range and below the target range were comparable during both study visits. However, median glucose on the second day of the closed-loop intervention was significantly reduced during the closed-loop period ( $p=0.027)$ which came at the expense of greater time spent in the hypoglycaemic range $(p=0.017)$.

Bihormonal systems have a high complexity and are presently limited by the need for a second pump device for glucagon delivery. The user is required to replace glucagon and the infusion set every $24 \mathrm{~h}$ due to the instability of current glucagon preparation during extended pump use [86]. Thus, efforts are underway to develop a dual-chamber pump device and stable glucagon preparation for use in bihormonal closedloop systems [87]. In addition, long-term data from human studies are needed to address the uncertainty regarding safety and tolerability associated with chronic subcutaneous glucagon.

\section{Other adjunctive approaches with closed-loop insulin delivery}

There is an increasing interest in the feasibility of adjunctive therapies to suppress postprandial hyperglucagonaemia and associated hyperglycaemia [88]. The adjunctive therapies include pramlintide and glucagon-like peptide-1, which have been evaluated in combination with closed-loop insulin delivery in research facility settings.

A small study compared closed-loop insulin delivery either alone or with subcutaneous pramlintide before meals, during two $24 \mathrm{~h}$ periods [89]. No premeal insulin boluses or meal announcements were provided during either visit. Overall, pramlintide co-delivery significantly reduced the postprandial time to peak plasma glucose $(p<0.0001)$, plasma glucose excursions $(p=0.006)$ and the meal-related area under the curve glucose excursion $(p=0.04)$ compared with closed-loop therapy alone. In another study, the use of either pramlintide or exenatide during closed-loop insulin delivery were compared with closed-loop insulin delivery alone, over $27 \mathrm{~h}$ [90].
Compared with closed-loop insulin delivery alone, coadministration of exenatide, but not pramlintide, led to a significantly greater reduction in glucose levels after lunch and dinner $(p<0.03$ and $p>0.05$, respectively). Interestingly, glucagon suppression was significantly greater with exenatide co-administration $(p<0.03)$ but not with pramlintide co-administration $(p>0.05)$ when compared with closed-loop insulin delivery alone. The investigators reported no increase in hypoglycaemia episodes with either exenatide or pramlintide. The number of participants who experienced gastrointestinal adverse events, however, was higher with exenatide (three participants experienced nausea, and one had an episode of vomiting) compared with pramlintide (one experienced nausea).

\section{Outlook and conclusions}

Evidence from transitional and home studies is encouraging, demonstrating progress towards real life closed-loop clinical use [91]. International and national funders have cumulatively provided grants in excess of $\$ 200$ million for closed-loop academic research over the past decade, whilst device manufacturers have committed significant resources towards commercialisation. For example, in the first half of 2016, a pivotal study of the hybrid closed-loop 670G insulin pump was completed by Medtronic. With a large amount of stakeholder engagement and a relatively low developmental risk there is an expectation for the technology to be available in clinical practice before the end of the decade. In line with this, a recent review by the UK National Institute for Health Research reported that automated closed-loop systems may be expected to appear in the market by the end of 2018 [92]. This will largely be dependent upon regulatory approvals (but there is a reassuring attitude of regulatory agencies such as the US Food and Drug Administration towards these therapies) and whether infrastructures and support are in place for the healthcare professionals providing clinical care. Structured education will also need to continue to augment efficacy and safety of this therapy. It is also important to note that, since closed-loop devices may be vulnerable to cybersecurity threats, such as interference with wireless protocols and unauthorised data retrieval [93], implementation of secure communications protocols is vital.

The cost-effectiveness of closed-loop systems is to be determined to support access and reimbursement to healthcare users and funders, respectively. In addition to the conventional endpoints, such as $\mathrm{HbA}_{1 \mathrm{c}}$, quality of life is to be included to assess the burden of disease management and associated hypoglycaemia. Future research may include elucidating the subpopulations which may benefit most and, as such, research 
is on-going to evaluate the efficacy of closed-loop systems in the very young [94], pregnant women with type 1 diabetes [95] and those with inpatient hyperglycaemia [96, 97]. Prolonged 6-24 month multinational closed-loop clinical trials are currently underway or in preparation, using adult and paediatric populations.

Technological advances in glucose sensing may provide marginal improvements in glucose management outcomes with the use of closed-loop systems, but may be a key driver for the adoption of closed-loop therapy by users if the size of the glucose sensor is reduced, sensor wear time prolonged and the need for calibration avoided. Further technological advancements should also focus on improvements in insulin delivery to prolong infusion catheter use, reduce silent infusion catheter occlusions and accelerate insulin absorption and action to improve efficacy of closed-loop therapies, possibly allowing for the development of a fully closed-loop system without the need for user-initiated prandial insulin dosing. Control algorithms play a crucial role in facilitating adaptation and individualisation whilst mitigating against imperfections of other closed-loop system components including glucose sensing inaccuracies and pump delivery errors. These adaptation capabilities are distinguishing features of closed-loop systems and, thus, advances in these technologies would also be of benefit for the efficacy of these devices.

Significant milestones, with research moving from laboratory to free-living unsupervised home settings, have been achieved in the past decade. Through inter-disciplinary collaboration, an accelerated progress in real world closedloop application has been demonstrated. Given the challenges of curative cell based and immunological therapies, closedloop technologies provide a viable alternative for pancreatic endocrine replacement therapy and have a continuing innovation potential.

Funding Supported by the National Institute of Health Research Cambridge Biomedical Research Centre, Efficacy and Mechanism Evaluation National Institute for Health Research (\#14/23/09), The Leona M. \& Harry B. Helmsley Charitable Trust (\#2016PG-T1D045), JDRF (\#2-SRA-2014-256-M-R), National Institute of Diabetes and Digestive and Kidney Diseases (1UC4DK108520-01) and Diabetes UK (\#14/0004878).

Duality of interest RH reports having received speaker honoraria from Eli Lilly and Novo Nordisk, serving on advisory panel for Eli Lilly and Novo Nordisk, receiving license fees from B. Braun and Medtronic; having served as a consultant to B. Braun, and patents and patent applications related to closed-loop. HT declares no duality of interest associated with this manuscript.

Contribution statement Both authors were responsible for drafting the article and revising it critically for important intellectual content. Both authors approved the version published.
Open Access This article is distributed under the terms of the Creative Commons Attribution 4.0 International License (http:// creativecommons.org/licenses/by/4.0/), which permits unrestricted use, distribution, and reproduction in any medium, provided you give appropriate credit to the original author(s) and the source, provide a link to the Creative Commons license, and indicate if changes were made.

\section{References}

1. Clarke SF, Foster JR (2012) A history of blood glucose meters and their role in self-monitoring of diabetes mellitus. Br J Biomed Sci 69:83-93

2. Pickup JC, Keen H, Parsons JA, Alberti KG (1978) Continuous subcutaneous insulin infusion: an approach to achieving normoglycaemia. Br Med J 1:204-207

3. Tamborlane WV, Sherwin RS, Genel M, Felig P (1979) Reduction to normal of plasma glucose in juvenile diabetes by subcutaneous administration of insulin with a portable infusion pump. $\mathrm{N}$ Engl J Med 300:573-578

4. Pickup JC (2012) Management of diabetes mellitus: is the pump mightier than the pen? Nat Rev Endocrinol 8:425-433

5. Tamada JA, Garg S, Jovanovic L, Pitzer KR, Fermi S, Potts RO (1999) Noninvasive glucose monitoring: comprehensive clinical results. Cygnus Research Team. JAMA 282:1839-1844

6. Garg SK, Potts RO, Ackerman NR, Fermi SJ, Tamada JA, Chase HP (1999) Correlation of fingerstick blood glucose measurements with GlucoWatch biographer glucose results in young subjects with type 1 diabetes. Diabetes Care 22:1708-1714

7. Rodbard D (2016) Continuous glucose monitoring: a review of successes, challenges, and opportunities. Diabetes Technol Ther 18(Suppl 2):S23-S213

8. Thabit H, Tauschmann M, Allen JM et al (2015) Home use of an artificial beta cell in type 1 diabetes. N Engl J Med 373:2129-2140

9. Peyser T, Dassau E, Breton M, Skyler JS (2014) The artificial pancreas: current status and future prospects in the management of diabetes. Ann N Y Acad Sci 1311:102-123

10. Hovorka R (2011) Closed-loop insulin delivery: from bench to clinical practice. Nat Rev Endocrinol 7:385-395

11. Ly TT, Nicholas JA, Retterath A, Lim EM, Davis EA, Jones TW (2013) Effect of sensor-augmented insulin pump therapy and automated insulin suspension vs standard insulin pump therapy on hypoglycemia in patients with type 1 diabetes: a randomized clinical trial. JAMA 310:1240-1247

12. Bergenstal RM, Klonoff DC, Garg SK et al (2013) Threshold-based insulin-pump interruption for reduction of hypoglycemia. N Engl J Med 369:224-232

13. Maahs DM, Calhoun P, Buckingham BA et al (2014) A randomized trial of a home system to reduce nocturnal hypoglycemia in type 1 diabetes. Diabetes Care 37:1885-1891

14. Kropff J, DeVries JH (2016) Continuous glucose monitoring, future products, and update on worldwide artificial pancreas projects. Diabetes Technol Ther 18(Suppl 2):S2-53-S2-63

15. Thabit H, Hovorka R (2014) Bringing closed- loop home: recent advances in closed- loop insulin delivery. Curr Opin Endocrinol Diabetes Obes 21:95-101

16. The Diabetes Control and Complications Trial Research Group (1993) The effect of intensive treatment of diabetes on the development and progression of long-term complications in insulin-dependent diabetes mellitus. N Engl J Med 329:977-986

17. Nathan DM, Cleary PA, Backlund JY et al (2005) Intensive diabetes treatment and cardiovascular disease in patients with type 1 diabetes. N Engl J Med 353:2643-2653 
18. Cryer PE (2008) The barrier of hypoglycemia in diabetes. Diabetes 57:3169-3176

19. Pickup JC, Sutton AJ (2008) Severe hypoglycaemia and glycaemic control in Type 1 diabetes: meta-analysis of multiple daily insulin injections compared with continuous subcutaneous insulin infusion. Diabet Med 25:765-774

20. Pedersen-Bjergaard U, Kristensen PL, Beck-Nielsen H et al (2014) Effect of insulin analogues on risk of severe hypoglycaemia in patients with type 1 diabetes prone to recurrent severe hypoglycaemia (HypoAna trial): a prospective, randomised, open-label, blinded-endpoint crossover trial. Lancet Diabetes Endocrinol 2:553-561

21. Ruan Y, Thabit H, Leelarathna L et al (2016) Variability of insulin requirements over 12 weeks of closed-loop insulin delivery in adults with type 1 diabetes. Diabetes Care 39:830-832

22. Bell KJ, Smart CE, Steil GM, Brand-Miller JC, King B, Wolpert HA (2015) Impact of fat, protein, and glycemic index on postprandial glucose control in type 1 diabetes: implications for intensive diabetes management in the continuous glucose monitoring era. Diabetes Care 38:1008-1015

23. Manohar C, Levine JA, Nandy DK et al (2012) The effect of walking on postprandial glycemic excursion in patients with type 1 diabetes and healthy people. Diabetes Care 35:2493-2499

24. Bally L, Zueger T, Buehler T et al (2016) Metabolic and hormonal response to intermittent high-intensity and continuous moderate intensity exercise in individuals with type 1 diabetes: a randomised crossover study. Diabetologia 59:776-784

25. Brown SA, Jiang B, McElwee-Malloy M, Wakeman C, Breton MD (2015) Fluctuations of hyperglycemia and insulin sensitivity are linked to menstrual cycle phases in women with type 1 diabetes. $\mathrm{J}$ Diabetes Sci Technol 9:1192-1199

26. Wong JC, Neinstein AB, Spindler M, Adi S (2015) A minority of patients with type 1 diabetes routinely downloads and retrospectively reviews device data. Diabetes Technol Ther 17:555-562

27. Beck RW (2015) Downloading diabetes device data: empowering patients to download at home to achieve better outcomes. Diabetes Technol Ther 17:536-537

28. Simmons JH, Chen V, Miller KM et al (2013) Differences in the management of type 1 diabetes among adults under excellent control compared with those under poor control in the T1D Exchange Clinic Registry. Diabetes Care 36:3573-3577

29. Hovorka R (2015) Artificial pancreas project at Cambridge 2013. Diabet Med 32:987-992

30. Johnson SR, Cooper MN, Davis EA, Jones TW (2013) Hypoglycaemia, fear of hypoglycaemia and quality of life in children with Type 1 diabetes and their parents. Diabet Med 30:11261131

31. Brod M, Wolden M, Christensen T, Bushnell DM (2013) A nine country study of the burden of non-severe nocturnal hypoglycaemic events on diabetes management and daily function. Diabetes Obes Metab 15:546-557

32. van Bon AC, Brouwer TB, von Basum G, Hoekstra JB, DeVries JH (2011) Future acceptance of an artificial pancreas in adults with type 1 diabetes. Diabetes Technol Ther 13:731-736

33. Elleri D, Acerini CL, Allen JM et al (2010) Parental attitudes towards overnight closed-loop glucose control in children with type 1 diabetes. Diabetes Technol Ther 12:35-39

34. Barnard KD, Pinsker JE, Oliver N, Astle A, Dassau E, Kerr D (2015) Future artificial pancreas technology for type 1 diabetes: what do users want? Diabetes Technol Ther 17:311-315

35. Wong JC, Foster NC, Maahs DM et al (2014) Real-time continuous glucose monitoring among participants in the T1D Exchange clinic registry. Diabetes Care 37:2702-2709

36. de Bock M, Cooper M, Retterath A et al (2016) Continuous glucose monitoring adherence: Lessons from a clinical trial to predict outpatient behavior. J Diabetes Sci Technol 10:627-632
37. Barnard KD, Wysocki T, Thabit $\mathrm{H}$ et al (2015) Psychosocial aspects of closed- and open-loop insulin delivery: closing the loop in adults with Type 1 diabetes in the home setting. Diabet Med 32:601-608

38. Ziegler C, Liberman A, Nimri R et al (2015) Reduced worries of hypoglycaemia, high satisfaction, and increased perceived ease of use after experiencing four nights of MD-Logic artificial pancreas at home (DREAM4). J Diabetes Res 2015:590308

39. Bruni A, Gala-Lopez B, Pepper AR, Abualhassan NS, Shapiro AJ (2014) Islet cell transplantation for the treatment of type 1 diabetes: recent advances and future challenges. Diabetes Metab Syndr Obes 7:211-223

40. Kelly WD, Lillehei RC, Merkel FK, Idezuki Y, Goetz FC (1967) Allotransplantation of the pancreas and duodenum along with the kidney in diabetic nephropathy. Surgery 61:827-837

41. Gruessner RW, Gruessner AC (2013) The current state of pancreas transplantation. Nat Rev Endocrinol 9:555-562

42. Gruessner AC, Gruessner RW (2012) Pancreas transplant outcomes for United States and non United States cases as reported to the United Network for Organ Sharing and the International Pancreas Transplant Registry as of December 2011. Clin Transpl:23-40

43. de Ferranti SD, de Boer IH, Fonseca V et al (2014) Type 1 diabetes mellitus and cardiovascular disease: a scientific statement from the American Heart Association and American Diabetes Association. Diabetes Care 37:2843-2863

44. Rudolph EN, Finger EB, Chandolias N, Kandaswamy R, Sutherland DE, Dunn TB (2015) Outcomes of pancreas retransplantation. Transplantation 99:367-374

45. Lakey JR, Burridge PW, Shapiro AM (2003) Technical aspects of islet preparation and transplantation. Transpl Int 16:613-632

46. Ryan EA, Paty BW, Senior PA et al (2005) Five-year follow-up after clinical islet transplantation. Diabetes 54:2060-2069

47. Shapiro AM (2011) State of the art of clinical islet transplantation and novel protocols of immunosuppression. Curr Diab Rep 11: 345-354

48. Phillip M, Battelino T, Atlas E et al (2013) Nocturnal glucose control with an artificial pancreas at a diabetes camp. N Engl J Med 368:824-833

49. Russell SJ, El-Khatib FH, Sinha M et al (2014) Outpatient glycemic control with a bionic pancreas in type 1 diabetes. N Engl J Med 371: 313-325

50. Leelarathna L, Nodale M, Allen JM et al (2013) Evaluating the accuracy and large inaccuracy of two continuous glucose monitoring systems. Diabetes Technol Ther 15:143-149

51. Thabit H, Leelarathna L, Wilinska ME et al (2015) Accuracy of continuous glucose monitoring during three closed-loop home studies under free-living conditions. Diabetes Technol Ther 17:801-807

52. Bergenstal RM, Tamborlane WV, Ahmann A et al (2010) Effectiveness of sensor-augmented insulin-pump therapy in type 1 diabetes. N Engl J Med 363:311-320

53. Choudhary P, Olsen BS, Conget I, Welsh JB, Vorrink L, Shin JJ (2016) Hypoglycemia prevention and user acceptance of an insulin pump system with predictive low glucose management. Diabetes Technol Ther 18:288-291

54. Steil GM, Rebrin K, Darwin C, Hariri F, Saad MF (2006) Feasibility of automating insulin delivery for the treatment of type 1 diabetes. Diabetes 55:3344-3350

55. Hovorka R, Canonico V, Chassin LJ et al (2004) Nonlinear model predictive control of glucose concentration in subjects with type 1 diabetes. Physiol Meas 25:905-920

56. Weinzimer SA, Steil GM, Swan KL, Dziura J, Kurtz N, Tamborlane WV (2008) Fully automated closed-loop insulin delivery versus semiautomated hybrid control in pediatric patients with type 1 diabetes using an artificial pancreas. Diabetes Care 31:934-939

57. El-Khatib FH, Russell SJ, Nathan DM, Sutherlin RG, Damiano ER (2010) A bihormonal closed-loop artificial pancreas for type 1 diabetes. Sci Transl Med 2:27ra27 
58. Haidar A, Elleri D, Kumareswaran K et al (2013) Pharmacokinetics of insulin aspart in pump-treated subjects with type 1 diabetes: reproducibility and effect of age, weight, and duration of diabetes. Diabetes Care 36:e173-e174

59. Home PD (2012) The pharmacokinetics and pharmacodynamics of rapid-acting insulin analogues and their clinical consequences. Diabetes Obes Metab 14:780-788

60. Garg SK, Voelmle M, Gottlieb PA (2010) Time lag characterization of two continuous glucose monitoring systems. Diabetes Res Clin Pract 87:348-353

61. Basu A, Dube S, Veettil S et al (2015) Time lag of glucose from intravascular to interstitial compartment in type 1 diabetes. $\mathrm{J}$ Diabetes Sci Technol 9:63-68

62. Heise T, Hövelmann U, Brøndsted L, Adrian CL, Nosek L, Haahr H (2015) Faster-acting insulin aspart: earlier onset of appearance and greater early pharmacokinetic and pharmacodynamic effects than insulin aspart. Diabetes Obes Metab 17:682-688

63. Setji TL, Hong BD, Feinglos MN (2015) Technosphere insulin: inhaled prandial insulin. Expert Opin Biol Ther:1-7

64. Zisser H, Dassau E, Lee JJ, Harvey RA, Bevier W, Doyle FJ (2015) Clinical results of an automated artificial pancreas using technosphere inhaled insulin to mimic first-phase insulin secretion. J Diabetes Sci Technol 9:564-572

65. El-Laboudi AH, Oliver N (2015) Towards a physiological prandial insulin profile: Enhancement of subcutaneously injected prandial insulin using local warming devices. Diabetes Ther 6:257-272

66. Wang X, Ioacara S, DeHennis A (2015) Long-term home study on nocturnal hypoglycemic alarms using a new fully implantable continuous glucose monitoring system in type 1 diabetes. Diabetes Technol Ther 17:780-786

67. Bailey T, Bode BW, Christiansen MP, Klaff LJ, Alva S (2015) The performance and usability of a factory-calibrated flash glucose monitoring system. Diabetes Technol Ther 17:787-794

68. Hajnsek M, Nacht B, Sax S, List EJ, Klimant I, Sinner F (2014) The single-port concept: combining optical glucose measurement with insulin infusion. Acta Diabetol 51:883-886

69. Del Favero S, Place J, Kropff J et al (2015) Multicenter outpatient dinner/overnight reduction of hypoglycemia and increased time of glucose in target with a wearable artificial pancreas using modular model predictive control in adults with type 1 diabetes. Diabetes Obes Metab 17:468-476

70. Del Favero S, Bruttomesso D, Di Palma F et al (2014) First use of model predictive control in outpatient wearable artificial pancreas. Diabetes Care 37:1212-1215

71. Grosman B, Ilany J, Roy A et al (2016) Hybrid closed-loop insulin delivery in type 1 diabetes during supervised outpatient conditions. J Diabetes Sci Technol 10:708-731

72. Ly TT, Breton MD, Keith-Hynes P et al (2014) Overnight glucose control with an automated, unified safety system in children and adolescents with type 1 diabetes at diabetes camp. Diabetes Care 37:2310-2316

73. Ly TT, Roy A, Grosman B et al (2015) Day and night closed-loop control using the integrated Medtronic hybrid closed-loop system in type 1 diabetes at diabetes camp. Diabetes Care 38:1205-1211

74. Kovatchev BP, Renard E, Cobelli C et al (2014) Safety of outpatient closed-loop control: first randomized crossover trials of a wearable artificial pancreas. Diabetes Care 37:1789-1796

75. Brown SA, Kovatchev BP, Breton MD et al (2015) Multinight "bedside" closed-loop control for patients with type 1 diabetes. Diabetes Technol Ther 17:203-209

76. Nimri R, Muller I, Atlas E et al (2014) MD-Logic overnight control for 6 weeks of home use in patients with type 1 diabetes: randomized crossover trial. Diabetes Care 37:3025-3032

77. Leelarathna L, Dellweg S, Mader JK et al (2014) Day and night home closed-loop insulin delivery in adults with type 1 diabetes: three-center randomized crossover study. Diabetes Care 37:19311937

78. Tauschmann M, Allen JM, Wilinska ME et al (2016) Day-and-night hybrid closed-loop insulin delivery in adolescents with type 1 diabetes: a free-living, randomized clinical trial. Diabetes Care. doi:10.2337/dc15-2078

79. Kropff J, Del Favero S, Place J et al (2015) 2 month evening and night closed-loop glucose control in patients with type 1 diabetes under free-living conditions: a randomised crossover trial. Lancet Diabetes Endocrinol 3:939-947

80. Barnard KD, Wysocki T, Allen JM et al (2014) Closing the loop overnight at home setting: psychosocial impact for adolescents with type 1 diabetes and their parents. BMJ Open Diabetes Res Care 2: e000025. doi:10.1136/bmjdrc-2014-000025

81. Bakhtiani PA, Zhao LM, El Youssef J, Castle JR, Ward WK (2013) A review of artificial pancreas technologies with an emphasis on bihormonal therapy. Diabetes Obes Metab 15:1065-1070

82. Haidar A, Smaoui MR, Legault L, Rabasa-Lhoret R (2016) The role of glucagon in the artificial pancreas. Lancet Diabetes Endocrinol 4: 476-479

83. Russell SJ, Hillard MA, Balliro C et al (2016) Day and night glycaemic control with a bionic pancreas versus conventional insulin pump therapy in preadolescent children with type 1 diabetes: a randomised crossover trial. Lancet Diabetes Endocrinol $4: 233-243$

84. Haidar A, Legault L, Matteau-Pelletier L et al (2015) Outpatient overnight glucose control with dual-hormone artificial pancreas, single-hormone artificial pancreas, or conventional insulin pump therapy in children and adolescents with type 1 diabetes: an openlabel, randomised controlled trial. Lancet Diabetes Endocrinol 3: 595-604

85. van Bon AC, Luijf YM, Koebrugge R, Koops R, Hoekstra JB, Devries JH (2014) Feasibility of a portable bihormonal closedloop system to control glucose excursions at home under freeliving conditions for 48 hours. Diabetes Technol Ther 16:131-136

86. Steiner SS, Li M, Hauser R, Pohl R (2010) Stabilized glucagon formulation for bihormonal pump use. J Diabetes Sci Technol 4: $1332-1337$

87. Jackson MA, Caputo N, Castle JR, David LL, Roberts CT, Ward WK (2012) Stable liquid glucagon formulations for rescue treatment and bi-hormonal closed-loop pancreas. Curr Diab Rep 12: $705-710$

88. Cryer PE (2012) Minireview: Glucagon in the pathogenesis of hypoglycemia and hyperglycemia in diabetes. Endocrinology 153 : $1039-1048$

89. Weinzimer SA, Sherr JL, Cengiz E et al (2012) Effect of pramlintide on prandial glycemic excursions during closed-loop control in adolescents and young adults with type 1 diabetes. Diabetes Care 35:1994-1999

90. Renukuntla VS, Ramchandani N, Trast J, Cantwell M, Heptulla RA (2014) Role of glucagon-like peptide-1 analogue versus amylin as an adjuvant therapy in type 1 diabetes in a closed loop setting with ePID algorithm. J Diabetes Sci Technol 8:1011-1017

91. Kowalski A (2015) Pathway to artificial pancreas systems revisited: moving downstream. Diabetes Care 38:1036-1043

92. Trevitt S, Simpson S, Wood A (2015) Artificial pancreas device systems for the closed-loop control of type 1 diabetes: what systems are in development? J Diabetes Sci Technol 10:714-723

93. O'Keeffe DT, Maraka S, Basu A, Keith-Hynes P, Kudva YC (2015) Cybersecurity in artificial pancreas experiments. Diabetes Technol Ther 17:664-666

94. Dauber A, Corcia L, Safer J, Agus MS, Einis S, Steil GM (2013) Closed-loop insulin therapy improves glycemic control in children aged $<7$ years: a randomized controlled trial. Diabetes Care 36: $222-227$ 
95. Murphy HR, Elleri D, Allen JM et al (2011) Closed-loop insulin delivery during pregnancy complicated by type 1 diabetes. Diabetes Care 34:406-411

96. Leelarathna L, English SW, Thabit H et al (2013) Feasibility of fully automated closed-loop glucose control using continuous subcutaneous glucose measurements in critical illness: a randomized controlled trial. Crit Care 17:R159

97. Thabit H, Hovorka R (2014) Glucose control in non-critically ill inpatients with diabetes: towards closed-loop. Diabetes Obes Metab 16:500-509 